\title{
Development of FPGA-Based Real-Time Neutron Spectrometer Using Stilbene Scintillator*)
}

\author{
Egor I. PINZHENIN, Aleksandr D. KHILCHENKO, Peter V. ZUBAREV, Andrey N. KVASHNIN, \\ Yury A. KASHCHUK ${ }^{1)}$, Andrey O. KOVALEV ${ }^{1)}$ and Sergey Yu. OBUDOVSKY ${ }^{1)}$ \\ Budker Institute of Nuclear Physics SB RAS, Novosibirsk 630090, Russia \\ ${ }^{1)}$ TRINITI, Moscow 142092, Russia
}

(Received 28 September 2018 / Accepted 18 December 2018)

\begin{abstract}
Neutron/gamma ray spectrometer based on a single-crystal stilbene scintillator with the photomultiplier tube 9266B was developed and tested. Signal acquisition and real-time processing is enabled by the fast ADC with the wide-band preamplifier and the Field-Programmable Gate Array (FPGA) core. The method of $n / \gamma$ event separation based on frequency gradient analysis was implemented. The paper shows the efficiency of $n / \gamma$ separation delivered by the spectrometer. The energy scale calibration of gamma channel was carried out by radionuclide gamma sources. The neutron channel energy calibration was done using accelerator-based neutron sources with deuterium-deuterium and deuterium-tritium fusion reactions. Real time registration of neutron spectra and gamma spectra in different channels of spectrometer was shown when acquiring amixed neutron and gamma flux. The paper illustrates the possibility of simultaneous registrations of $2.45 \mathrm{MeV}$ and $14 \mathrm{MeV}$ neutrons. The energy resolution of neutron spectrometer was measured. The neutron count rate up to $210^{5} \mathrm{~s}^{-1}$ was demonstrated.
\end{abstract}

(C) 2019 The Japan Society of Plasma Science and Nuclear Fusion Research

Keywords: stilbene scintillator, neutron spectrometry, frequency gradient analysis

DOI: $10.1585 /$ pfr. 14.2402025

\section{Introduction}

Neutron diagnostic is an important tool for determining fusion power, power density and the plasma ion temperature in experiments with hot deuterium and tritium plasmas [1]. Furthermore the neutron spectrometer is an ultimate instrument for determining the relative power released in each of the reactions (DD reaction and DT reaction) in the forthcoming experiments with DT plasma. The concentration of deuterium and tritium in the plasma can be estimated using this technique. Thus, it is important to have a neutron spectrometer at an energy range from $1 \mathrm{MeV}$ to $15 \mathrm{MeV}$.

Contribution to the signal provided by the gamma ray emission from the plasma formed, for example, as a result of $(n, \gamma)$ reactions, is an additional complication in neutron spectroscopy experiments using scintillator based detectors. One of the methods of reducing this unwanted contribution to measurements is to use organic scintillator like stilbene, where the shape of the light flash depends on the type of registered particle (Fig. 1). Namely, light pulses caused by neutrons, alpha particles and gamma rays have different decay times in stilbene, p-terphenyl and some liquid scintillators (NE213, BC501). Accordingly, the energy spectra for all these particles can be analyzed independently applying event separation on the basis of light

author's e-mail: e.i.pinzhenin@inp.nsk.su

*) This article is based on the presentation at the 12th International Conference on Open Magnetic Systems for Plasma Confinement (OS2018).

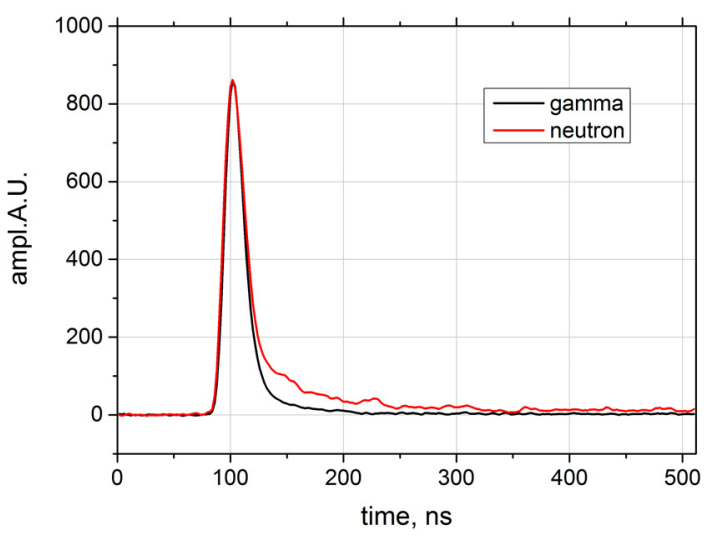

Fig. 1 Gamma ray and neutron pulses recorded with the detector based on astilbene scintillator. The data for gamma rays was averaged over 10 events (fast decay time $\sim 6 \mathrm{~ns}$ ), the data for neutrons was averaged over 2 events (slow decay time $\sim 80 \mathrm{~ns}$ ).

pulse shape [2-4].

\section{The Neutron Spectrometer Design Overview}

The neutron spectrometer based on a single-crystal stilbene scintillator (diameter $30 \mathrm{~mm}$, length $30 \mathrm{~mm}$ ) was developed in the laboratory. This spectrometer uses the PMT 9266B for light burst detection, the fast preamplifier 


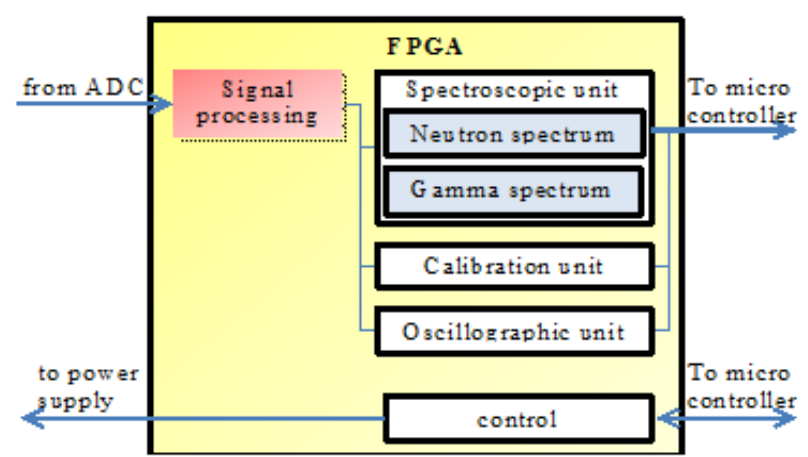

Fig. 2 Scheme of FPGA operation.

and the specially designed signal recording module. The latter is comprised of the ADC with the amplitude resolution of 12 bit and the sampling rate of $500 \mathrm{MSPS}$, and the digital processing core build in the FPGA. Such a spectrometer operates in real time with separation of neutron and gamma events. The frequency gradient analysis [5] is implemented for separation of events and the error in the separation method is less than the one for methods based on pulse shape analysis.

Several stages of the operation sequence were realized in the FPGA (Fig. 2). The oscillographic unit of the device is used for registering 16 events, each consisting of 256 counts at the sampling frequency of $500 \mathrm{MHz}$. It is used for determine the trigger level, to review the pulse shape and the noise level. Events with the amplitude less than the trigger level are not processed. Figure 1 shows the example of obtained data.

The calibration unit is used for determining the condition of separation. For that, 2048 events must be registered. Firstly, the base line of the signal is eliminated. The essence of the method is that high-frequency harmonics of the signal are the same, but low-frequency harmonics are different for neutrons and gamma quants. On the next stage, the frequency gradient analysis method [5] is used and $X(0)$ and $X(2)$ values arecalculated for each event.

$$
\begin{aligned}
& X(0)=\sum_{n=0}^{N-1} x(n)=N \bar{x}, \\
& X(2)=\sqrt{\left|X(2)_{\mathrm{re}}\right|^{2}+\left|X(2)_{\mathrm{im}}\right|^{2}},
\end{aligned}
$$

where

$$
\begin{aligned}
& X(2)_{\mathrm{re}}=\sum_{n=0}^{N-1} x(n) \cos \left(2 * \frac{2 \pi}{N} n\right), \\
& X(2)_{\mathrm{im}}=\sum_{n=0}^{N-1} x(n) \sin \left(2 * \frac{2 \pi}{N} n\right),
\end{aligned}
$$

$X(0)$ and $X(2)$ are magnitudes of Discrete Fourier Transform applied to the registered signal. The number $N$ is the signal length, $x(n)$ is the measured value at the time point $n$. Then the values $X(0) / X(2)$ are calculated. The bar graph

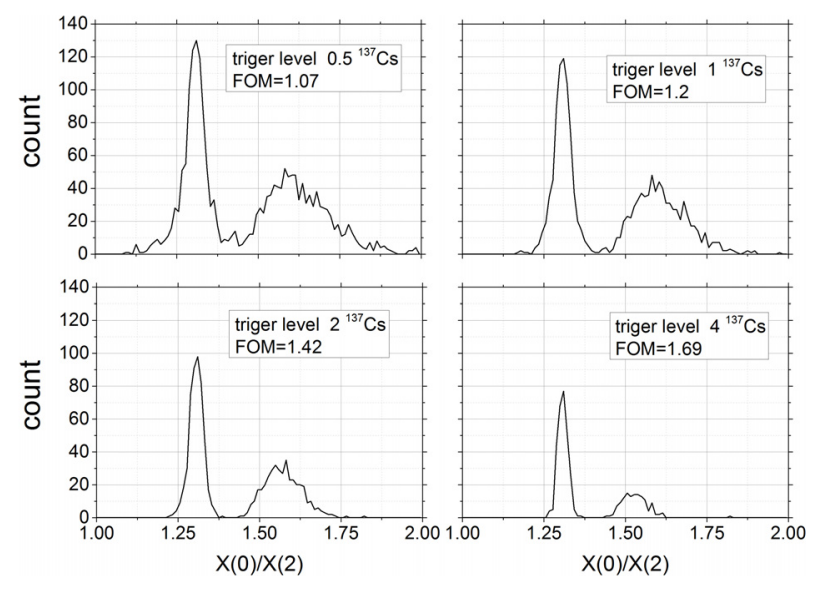

Fig. $3 X(0) / X(2)$ histogram for different trigger level. ${ }^{252} \mathrm{Cf}$ radionuclide source was used.

of those values is shown in the Fig. 3. The graph has two maximums if spectrometer registered a mixed neutron and gamma flux. Thereby if the measured value $X(0) / X(2)$ of some event is appeared to be greater than the certain preset number, the event is qualified as a neutron (right maximum in bar graph). Otherwise, it is qualified as a gamma ray (left maximum in bar graph).

Determination of the best parameter of separation is performed offline by the researcher. The FPGA program receives this setting from the input in the control interface of the spectrometer. The separation criterion was assumed 1.4 in all experiments shown in the paper.

The bar graph of $X(0) / X(2)$ is used for determining the efficiency of $n / \gamma$ separation. The FOM (Figure of Merit) valueis a measure of the separation efficiency.

$$
F O M=\frac{\Delta_{\text {peak }}}{F W H M_{\mathrm{n}}+F W H M_{\gamma}},
$$

where $F W H M_{\mathrm{n}}$ is the full-width half-maximum of the spread of neutron events, $F W H M_{\gamma}$ is the full-width halfmaximum of the spread of gamma events, $\Delta_{\text {peak }}$ is the distance between the peaks of neutron and gamma distribution. The separation reveals more prominently when the trigger threshold is increased. This condition is illustrated in the Fig. 3.

The spectroscopic unit is used for spectra registration. The values of $X(0)$ and $X(2)$ are calculated by the signal processing unit in the FPGA, as described above. If the value $X(0) / X(2)$ exceeds the criterion of separation (it is equals to 1.4 in discussed experiments), this event is determined as a neutron and it is recorded to the neutron channel. Otherwise, the given event is determined as a gamma ray and it is recorded to the spectra of gamma channel. All calculations are performed in real time and spectra registration iscarried out in real time regime as well. The count rate up to $210^{5} \mathrm{~s}^{-1}$ was demonstrated when mixed $n / \gamma$ flux is registered. 


\section{Energy Scale Calibration}

We used radionuclide gamma sources for energy scale calibration of the gamma channel of the spectrometer. Table 1 presents used radionuclide sources, the energy of gamma lines, the energy of Compton electrons and the identifier of the marker in the Fig. 4. Measured spectra are presented in the Fig. 4 (top plot). The photo effect peak is absent in measured gamma spectra because the efficiency of Compton effect is much greater than the efficiency of photo effect in a stilbene scintillator. Thus energy calibration was carried out at the Compton edge of gamma ray lines of radionuclide gamma sources. The maximum en-

Table 1 Gamma sources.

\begin{tabular}{cccc}
$\boldsymbol{\gamma}$ source & $\mathbf{E}_{\gamma}$ & $\mathbf{E}_{\max }$ & Marker No \\
\hline${ }^{22} \mathrm{Na}$ & $511 \mathrm{keV}$ & $340.4 \mathrm{keV}$ & 1 \\
${ }^{22} \mathrm{Na}$ & $1275 \mathrm{keV}$ & $1061.8 \mathrm{keV}$ & 4 \\
${ }^{137} \mathrm{Cs}$ & $662 \mathrm{keV}$ & $477 \mathrm{keV}$ & 2 \\
${ }_{60} \mathrm{Co}$ & $1173 \mathrm{keV}$ & $962.9 \mathrm{keV}$ & 3 \\
${ }_{60} \mathrm{Co}$ & $1333 \mathrm{keV}$ & $1118.2 \mathrm{keV}$ & 5 \\
$\mathrm{H} \mathrm{capture}$ & $2223 \mathrm{keV}$ & $1993.4 \mathrm{keV}$ & 6 \\
$\mathrm{AmBe}$ & $3927 \mathrm{keV}$ & $3687.1 \mathrm{keV}$ & 7 \\
$\mathrm{AmBe}$ & $4438 \mathrm{keV}$ & $4196.4 \mathrm{keV}$ & 8
\end{tabular}

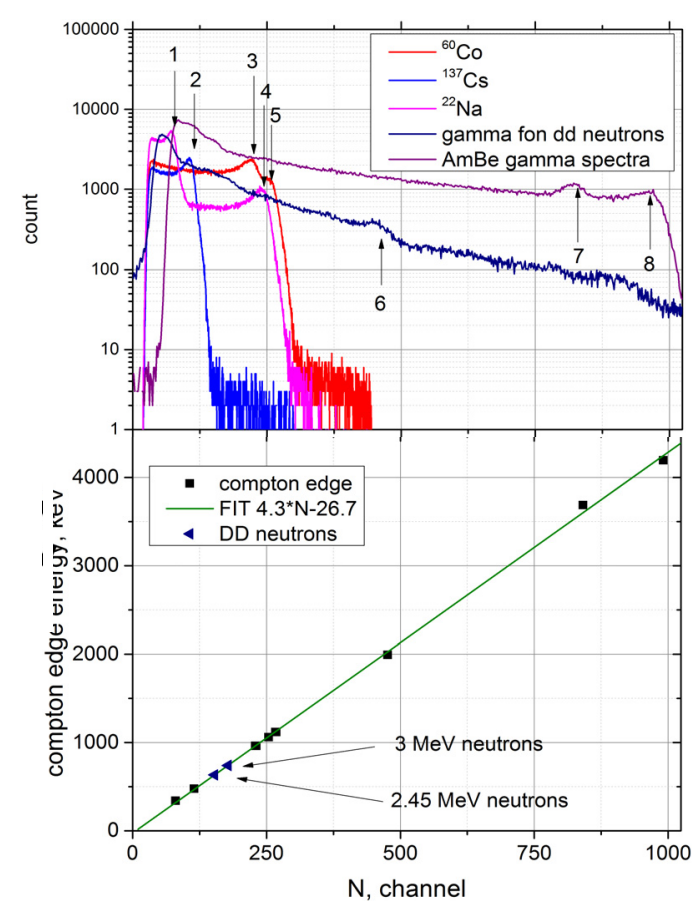

Fig. 4 The spectrum of gamma sources (top plot) and energy calibration (bottom plots). All data measured with the PMT voltage of $550 \mathrm{~V}$. The figure also presents data for neutrons with energy $2.45 \mathrm{MeV}$ and $3 \mathrm{MeV}$. ergy of Compton electrons $E_{\max }[\mathrm{keV}]$ is given by

$$
E_{\max }=E_{\gamma}\left(\frac{2 \gamma}{1+2 \gamma}\right),
$$

where $\gamma=E_{\gamma} / 511 \mathrm{keV}$ and $E_{\gamma}$ is the incident photon energy in keV. The Compton edge energy and Fig. 4 marker identifiers are presented in the Table 1.

We also used the AmBe source of $n / \gamma$ flux for energy calibration of the gamma channel (energies of $3927 \mathrm{keV}$ and $4438 \mathrm{keV}$ ). A polyethylene neutron moderator was used for obtain $2223 \mathrm{keV}$ gamma line of neutron capture by hydrogen. The particle accelerator based of $2.45 \mathrm{MeV}$ neutron source was used in this case.

The chosen calibration line for the gamma channel of the spectrometer is presented in the Fig. 4 (bottom plot). The PMT voltage of $550 \mathrm{~V}$ was set in this series of experiments. Data points of $2.45 \mathrm{MeV}$ and $3 \mathrm{MeV}$ neutron registrations are shown in this graph in addition.

Accelerated type neutron generators of DD fusion neutrons (ING-07DM, $10^{7}$ neutrons per sec [6]) and DT fusion neutrons (ING-07T, $10^{9}$ neutrons per sec [6]) were used for the energy scale calibration of the neutron channel of the spectrometer. Also the AmBe neutron source was used in this case. Spectra of $2.45 \mathrm{MeV}$ neutrons, $14 \mathrm{MeV}$ neutrons and the continuum spectrum of AmBe neutron source are presented in the Fig. 5. Moreover, the possibility of simultaneous registration of DD and DT neutrons

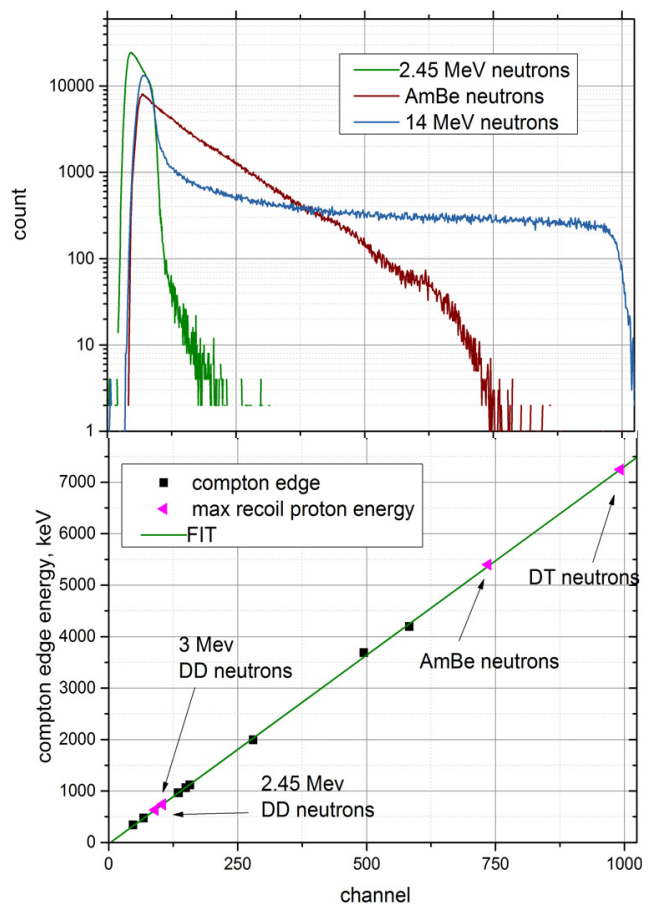

Fig. 5 The spectrum of DD, DT and AmBe neutron sources (top plot). Magenta dots shows the energy calibration for neutron sources (bottom plots). All data measured with $474 \mathrm{~V}$ of the PMT voltage. Black dots correspond to the data of Compton edges for gamma sources. 


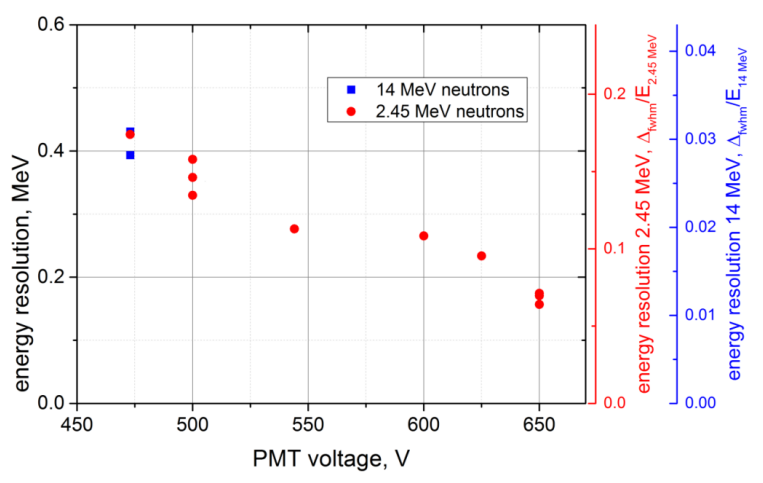

Fig. 6 Energy resolution of stilbene spectrometer. Data points for $2.45 \mathrm{MeV}$ and $14 \mathrm{MeV}$ neutrons are shown.

is demonstrated in the experiment in with neutron generator ING-07DM and neutron generator ING-07T operate together (see Fig. 5, blue line). The PMT voltage was $475 \mathrm{~V}$ in this setup.

The calibration line is presented in the Fig. 5 (bottom plot). Magenta triangle markers correspond to the maximal energy of recoil protons for each neutron source. In addition, the data of gamma channel calibration is presented. The difference of the recoil proton energy and the Compton electron energy is caused by the difference of light output of stilbene scintillator for particles of different species.

The DD neutron source of use produces neutrons with the energy spectrum from $2 \mathrm{MeV}$ to $3 \mathrm{MeV}$. The acceleration voltage was $150 \mathrm{keV}$, so the neutron energy to the detector direction was $2.45 \mathrm{MeV}$ for the spectrometer installed in the plane of target. For the spectrometer installed at the deuteron beam axis, the energy of measured neutron was $3 \mathrm{MeV}$. Both energies were used for the spectrometer calibration.

\section{Energy Resolution}

Energy resolution of neutron spectrometer depends on the PMT voltage and the energy of registered particle. Recoil proton spectrum measured in each experiment was differentiated. Energy resolution of the neutron spectrometer was assumed as the FWHM of the resulting distribution (in $\mathrm{keV}$ ) or FWHM divided to by the mean energy: $F W H M / E_{\text {mean }} * 100$ in percent. The Fig. 6 shows the energy resolution of the detector for different PMT voltage for $2.45 \mathrm{MeV}$ neutrons. The energy resolution for $14 \mathrm{MeV}$ neutrons was also measured. The corresponding data is presented in the Fig. 6 by blue points.

\section{Conclusion}

The neutron spectrometer based on a single-crystal stilbene (diameter $30 \mathrm{~mm}$, height $24 \mathrm{~mm}$ ), PMT 9266B,
ADC (12 bit, 500 MSPS) and FPGA was developed. The efficient neutron/gamma event separation algorithm based on the frequency-domain gradient analysis was implemented. The device operates in a real time regime. The count rate of up to $210^{5} \mathrm{~s}^{-1}$ was demonstrated when registering mixed neutron and gamma fluxes.

The detector registers the spectrum of recoil protons in the neutron channel and the spectrum of Compton electrons in the gamma channel separately.

The energy scale calibration was carried out using radioisotope gamma-ray sources $\left({ }^{137} \mathrm{Cs},{ }^{60} \mathrm{Co},{ }^{22} \mathrm{Na}\right)$, radioisotope neutron sources $\left({ }^{252} \mathrm{Cf}, \mathrm{AmBe}\right)$ and the acceleration type neutron generator of DD (ING-07DM [6]) and DT (ING-07T [6]) neutrons. Measurements were performed in a wide range of PMT operation parameters for neutrons from $2.45 \mathrm{MeV}$ to $14 \mathrm{MeV}$ and gamma rays from $500 \mathrm{keV}$ to $5 \mathrm{MeV}$. Simultaneous registration of DD and DT neutron spectra from neutron generators was demonstrated.

The instrument resolution of $180 \mathrm{keV}(7.3 \%)$ was obtained for $2.45 \mathrm{MeV}$ neutrons (the PMT voltage was $650 \mathrm{~V})$. The instrument resolution of $400 \mathrm{keV}(3 \%)$ was obtained for $14 \mathrm{MeV}$ neutrons (the PMT voltage was $475 \mathrm{~V})$.

The efficiency of neutron and gamma event separation increases with increasing of the threshold level of registration. The value of FOM = 1.2 was obtained for the detection threshold of $1.5 \mathrm{MeV}$ recoil protons and $477 \mathrm{keV}$ Compton electrons (corresponds to ${ }^{137} \mathrm{Cs}$ gamma-rays). Events corresponding to neutrons and gamma rays are separated reliably in this case.

Pilot measurements of neutron spectra and neutron fluxes from plasmas confined in the gas dynamic trap (GDT [7]) linear magnetic system were carried out by the stilbene based neutron spectrometer. The contribution of background gamma-rays into the measured spectrum is completely suppressed. The registered spectrum corresponds to $2.45 \mathrm{MeV}$ neutrons which are produced as a result of fusion DD reaction in GDT plasmas.

[1] L. Bertalot et al., J. Instrumentation 7, C04012 (2012).

[2] F.D. Brooks, Nucl. Instrum. Methods 4, Iss. 3, 151 (1959).

[3] M. Moszynski et al., Nucl. Instrum. Methods Phys. Res. A 317, Iss. 1-2, 262 (1992).

[4] A.A. Ivanova et al., Nucl. Instrum. Methods Phys. Res. A 827, 13 (2016).

[5] G. Liu et al., IEEE Trans. Nucl. Sci. 57, Iss. 3, part 3, 1682 (2010).

[6] D. Chernikova et al., Nucl. Instrum. Methods Phys. Res. A 746, 74 (2014).

[7] A.A. Ivanov and V.V. Prikhodko, Physics-Uspekhi 60, Iss. 5, 509 (2017). 